PHYSICAL REVIEW D 72, $104002(2005)$

\title{
Quantum gravity, torsion, parity violation, and all that
}

\author{
Laurent Freidel, ${ }^{1,2, *}$ Djordje Minic, ${ }^{3, \dagger}$ and Tatsu Takeuchi ${ }^{3, \ddagger}$ \\ ${ }^{1}$ Perimeter Institute for Theoretical Physics, 31 Caroline St. N. Waterloo, N2L 2Y5, Ontario, Canada \\ ${ }^{2}$ Laboratoire de Physique, Ecole Normale Supérieure de Lyon, 46 Allée d'Italie, 69364 Lyon, Cedex 07, France \\ ${ }^{3}$ Institute for Particle Physics and Astrophysics, Department of Physics, Virginia Tech, Blacksburg, Virginia 24061, USA
}

(Received 16 September 2005; published 1 November 2005)

\begin{abstract}
We discuss the issue of parity violation in quantum gravity. In particular, we study the coupling of fermionic degrees of freedom in the presence of torsion and the physical meaning of the Immirzi parameter from the viewpoint of effective field theory. We derive the low-energy effective Lagrangian which turns out to involve two parameters: one measuring the nonminimal coupling of fermions in the presence of torsion, the other being the Immirzi parameter. In the case of nonminimal coupling the effective Lagrangian contains an axial-vector interaction leading to parity violation. Alternatively, in the case of minimal coupling there is no parity violation and the effective Lagrangian contains only the usual axial-axial interaction. In this situation the real values of the Immirzi parameter are not at all constrained. On the other hand, purely imaginary values of the Immirzi parameter lead to violations of unitarity for the case of nonminimal coupling. Finally, the effective Lagrangian blows up for the positive and negative unit imaginary values of the Immirzi parameter.
\end{abstract}

DOI: 10.1103/PhysRevD.72.104002

PACS numbers: 04.60.Ds

\section{QUANTUM GRAVITY AND EFFECTIVE FIELD THEORY}

The Wilsonian point of view on effective field theory is a very powerful organizing principle allowing us to drastically restrict the number of theories relevant for the description of low-energy physics. As far as the low-energy physics is concerned, the Wilsonian approach instructs us to consider the most general local actions invariant under a given symmetry, while forgetting about the underlying microscopic degrees of freedom. Of course this philosophy might have to be radically changed in the presence of gravity, but in this paper we adopt the usual point of view in order to understand better the issue of gravitationally induced parity violation.

Gravity is a theory which is classically invariant under the diffeomorphism group and since we want to be able to incorporate standard model-like fermions, the effective field theory also should be invariant under a local Lorentz gauge symmetry. The dynamical field variables are given by the frame field (a one form valued in the vectorial representation) $e_{\mu}^{I} d x^{\mu}$ and Lorentz connection (a one form valued in the Lorentz Lie algebra) $\omega_{\mu}^{I J} d x^{\mu}$. If we apply the effective field theory point of view to gravity, and consider only actions which are analytic functionals of the frame field connection and its derivatives, ${ }^{1}$ we easily read out the most relevant terms in the low-energy effective action. To leading order this low-energy effective action contains exactly six possible terms. Three of these terms are topological invariants analogous to the QCD theta term.

\footnotetext{
*Electronic address: lfreidel@perimeterinstitute.ca

†Electronic address: dminic@ vt.edu

${ }^{\ddagger}$ Electronic address: takeuchi@vt.edu

${ }^{1}$ This excludes functionals which depend on the inverse frame field.
}

They correspond to the Euler class

$$
\theta_{1} \int R^{I J}(\omega) \wedge R^{K L}(\omega) \epsilon_{I J K L}
$$

the Pontryagin class

$$
\theta_{2} \int R^{I J}(\omega) \wedge R_{I J}
$$

and the Nieh-Yan class

$$
\theta_{3} \int d_{\omega} e^{I} \wedge d_{\omega} e_{I}-R^{I J} \wedge e_{I} \wedge e_{J}
$$

where $R^{I J}(\omega)=d \omega^{I J}+\omega^{I K} \wedge \omega_{K}^{J}$ is the canonical curvature two form. Since we are interested in semiclassical physics we concentrate our attention on the bulk terms. ${ }^{2}$ Two of the bulk terms are well known; they correspond to the Einstein-Hilbert action and the cosmological constant term. The corresponding coupling constants are, respectively, the Newton constant $G$ and the cosmological constant $\Lambda$. This is, however, not the final answer, for there exists an additional term which is usually disregarded. From an effective field theory point of view, this term is as important as the two others, and thus has to be taken into account in the low-energy effective action. The corresponding coupling constant is called the Immirzi parameter, $\gamma$.

Therefore, the leading bulk terms in the most general four-dimensional low-energy gravitational action, in any microscopic quantum theory of gravity (be it string theory, loop gravity etc.) are given by

\footnotetext{
${ }^{2}$ Topological "deformations" of Einstein's classical theory in four dimensions have been investigated in detail in [1].
} 


$$
\begin{aligned}
\tilde{S}_{P}= & -\frac{1}{32 \pi G} \int\left(R^{I J}(\omega) \wedge e^{K} \wedge e^{L} \epsilon_{I J K L}-\frac{\Lambda}{6} e^{I} \wedge e^{J}\right. \\
& \left.\wedge e^{K} \wedge e^{L} \epsilon_{I J K L}-\frac{2}{\gamma} R^{I J}(\omega) \wedge e_{I} \wedge e_{J}\right) .
\end{aligned}
$$

Note that unlike the Newton and cosmological constant, the Immirzi parameter is dimensionless. This action and the meaning of the Immirzi parameter was first discussed by Holst [2]. Usually $\gamma$ is taken to be equal to zero; this forces the torsion to be the null ${ }^{3}$ and we recover the usual second order formulation of gravity. It is also frequent but less common to encounter the choice $\gamma=\infty$. In this case we recover the Cartan-Weyl formulation of gravity [3].

From the effective field theory point of view there is $a$ priori no reason to prefer one choice or another, and thus, one should take into account all possible values of $\gamma$ and let experiment decide its numerical value. This is the point of view which is taken here. Effectively, at the quantum level, this amounts to treating $\gamma$ as a superselection parameter. We should add a caveat here: as briefly mentioned above, in this paper we assume the usual local Wilsonian effective theory, in which decoupling between the UV and IR degrees of freedom is usually taken for granted. This nevertheless does not have to be the case in the presence of gravity [4], but we refrain from any further discussion of this important issue in this paper [5].

Classically when there is no matter coupled to gravity the equations of motion for the connection imply zero torsion irrespective of the value of the Immirzi parameter. At the quantum level this is not the case and one expects observable effects associated with a particular microscopic formulation of quantum gravity (such as loop quantum gravity) which contains this parameter [6,7]. In [6] it is shown that $\gamma$ controls the rate of fluctuation of the torsion at the quantum level and when different from 0 or $\infty$, it leads to a compactification of the phase space of gravity. The Immirzi parameter plays an important role in loop quantum gravity, which predicts, for example, that the area of a surface or the volume of a spatial region are quantized [8] (the unit quanta of area and volume being $\gamma l_{P}^{2}$ and $\gamma^{3 / 2} l_{P}^{3}$ ). The spectra of the area and volume operators are discrete provided that the Immirzi parameter is not equal to zero. In contrast, there is no indication that area or volume are quantized in string theory (which might be because of the subtle issue of background independence). Thus any direct experimental constraint of the Immirzi parameter can be taken as concrete steps towards the falsification of a specific microscopic approach. This is the main motivation for the analysis that follows.

\footnotetext{
${ }^{3}$ The action (4) is singular when $\gamma=0$. One can however introduce an auxiliary field $B^{I}$ (a two form valued in the vectorial representation) in order to rewrite the Immirzi term, up to a boundary contribution, as $\int B^{I} \wedge T_{I}+\gamma T^{I} \wedge T_{I}$, where $T^{I}=$ $d_{\omega} e^{I}$ is the torsion. When $\gamma=0$ the equation of motion for the $B$ field forces the torsion to be null.
}

\section{TORSION AND FERMIONS}

In this section we follow the derivation of Rovelli and Perez [7], obtaining similar but crucially different results. We start with the formulation of general relativity in the first order formalism in terms of a Lorentz connection $\omega_{\mu}{ }^{I J}$ and a frame field $e_{\mu}^{I}$, where $I, J \ldots=0,1,2,3$ denotes the internal Lorentz indices and $\mu, \nu \ldots=0,1,2,3$ the respective space-time indices. The curvature is defined to be $R_{\mu \nu}^{I J}(\omega)=\partial_{\mu} \omega_{\nu}^{I J}-\partial_{\nu} \omega_{\mu}^{I J}+\left[\omega_{\mu}, \omega_{\nu}\right]^{I J}$. When the frame field is invertible the gravitational action (4) which includes the Immirzi parameter and a zero cosmological constant can be written in the form

$$
S_{G}[e, \omega]=\frac{1}{16 \pi G} \int d^{4} x e e_{I}^{\mu} e_{J}^{\nu} P_{K L}^{I J} R_{\mu \nu}^{K L}(\omega),
$$

where we have introduced the following tensor and its inverse

$$
\begin{aligned}
P^{I J}{ }_{K L} & =\delta_{K}^{[I} \delta_{L}^{J]}-\frac{1}{\gamma} \frac{\epsilon^{I J}{ }_{K L}}{2}, \\
P^{-1}{ }_{K L}{ }^{I J} & =\frac{\gamma^{2}}{\gamma^{2}+1}\left(\delta_{K}^{[I} \delta_{L}^{J]}+\frac{1}{\gamma} \frac{\epsilon^{I J}{ }_{K L}}{2}\right) .
\end{aligned}
$$

The coupling of gravity to fermions is given by the action

$$
S_{F}[e, \omega, \psi]=\int d^{4} x \frac{i e}{2}\left(\bar{\psi} \gamma^{I} e_{I}^{\mu} \nabla_{\mu} \psi-\overline{\nabla_{\mu} \psi} \gamma^{I} e_{I}^{\mu} \psi\right),
$$

where $\gamma^{I}$ are the Dirac matrices, ${ }^{4}$ and

$$
\nabla_{\mu} \equiv \partial_{\mu}+\omega_{\mu}^{I J} \frac{\gamma_{[I} \gamma_{J]}}{4}, \quad\left[\nabla_{\mu}, \nabla_{\nu}\right]=R_{\mu \nu}^{I J} \frac{\gamma_{[I} \gamma_{J]}}{4}
$$

We can decompose $\omega_{\mu}^{I J}$ as $\omega_{\mu}^{I J}=\widetilde{\omega}_{\mu}^{I J}+C_{\mu}^{I J}$, where $\tilde{\omega}$ is the torsion free spin connection satisfying

$$
\tilde{\nabla}_{[\mu} e_{\nu]}^{I}=0,
$$

and $C_{\mu}{ }^{I J}$ is the so called "contorsion" tensor related to the torsion in the obvious way $\nabla_{[\mu} e_{\nu]}^{I}=C_{[\mu}^{I J} e_{\nu] J}$.

It is important to consider in the fermion action the above real combination (a point overlooked in [7]) in order to get the same equation of motion for $\psi$ and $\bar{\psi}$. The general fermion action written in terms of the covariant derivative $\nabla$, which gives back the usual fermion action in the absence of gravity, can be written in the form

$$
\begin{aligned}
\tilde{S}_{F}[e, \omega, \psi]= & \int d^{4} x \frac{i e}{2}\left((1-i \alpha) \bar{\psi} \gamma^{I} e_{I}^{\mu} \nabla_{\mu} \psi\right. \\
& \left.-(1+i \alpha) \overline{\nabla_{\mu} \psi} \gamma^{I} e_{I}^{\mu} \psi\right) .
\end{aligned}
$$

Such an action does not give the same equation of motion

\footnotetext{
${ }^{4}$ We use the particle physics conventions $\left\{\gamma^{I}, \gamma^{J}\right\}=2 \eta^{I J}$ with $\eta^{I J}=\operatorname{diag}(+1,-1,-1,-1), \gamma_{5}=\gamma^{5}=i \gamma^{0} \gamma^{1} \gamma^{2} \gamma^{3}$. The reality conditions are $\gamma_{I}^{\dagger}=\gamma_{0} \gamma_{I} \gamma_{0},\left(i \gamma_{5}\right)^{\dagger}=\gamma_{0}\left(i \gamma_{5}\right) \gamma_{0}$.
} 
for $\psi$ and $\bar{\psi}$ unless

$$
\left(\alpha-\alpha^{*}\right) e_{I}^{\mu} C_{\mu}^{I J}=0 .
$$

If $\alpha$ is not real this imposes a constraint on the torsion tensor which is incompatible with the gravitational equation of motion that fixes the torsion. This is due to the fact that the following symmetric combination is not a total derivative in the presence of torsion [3]

$$
\begin{gathered}
\frac{e}{2}\left(\bar{\psi} \gamma^{I} e_{I}^{\mu} \nabla_{\mu} \psi+\overline{\nabla_{\mu} \psi} \gamma^{I} e_{I}^{\mu} \psi\right) \\
=\partial_{\mu}\left(e e_{I}^{\mu} V^{I}\right)+e_{I}^{\mu} C_{\mu J}^{I} V^{J},
\end{gathered}
$$

where $V^{I}$ is the vector fermion current ( $A^{I}$ denotes the axial fermion current)

$$
V^{I}=\bar{\psi} \gamma^{I} \psi, \quad A^{I}=\bar{\psi} \gamma^{5} \gamma^{I} \psi
$$

Both $V^{I}$ and $A^{I}$ are real currents. The value $\alpha=0$ corresponds to the usual minimal coupling of fermions to gravity. In general, an arbitrary real value for $\alpha$ corresponds to a nonminimal coupling.

We now consider the equation of motion coming from the variation of Eq. (7) with respect to the connection $\delta\left(S_{G}+S_{F}\right) / \delta \omega=0$. Since

$$
\frac{\delta S_{G}}{\delta \omega_{\mu}^{I J}}=\frac{1}{8 \pi G} \nabla_{\nu}\left(e e_{K}^{\mu} e_{L}^{\nu}\right) P_{I J}^{K L},
$$

and

$$
\frac{\delta S_{F}}{\delta \omega_{\mu}^{I J}}=\frac{i e e_{K}^{\mu}}{8} \bar{\psi}\left\{\gamma_{K}, \gamma_{[I} \gamma_{J]}\right\} \psi
$$

this equation of motion reads ${ }^{5}$

$$
\frac{1}{2 \pi G}\left(C_{[K L]}^{\mu}+C_{\nu[K}{ }^{\nu} e_{L]}^{\mu}\right) P_{I J}^{K L}=\epsilon_{I J}{ }^{K L} e_{K}^{\mu} A_{L}
$$

The solution is given by

$$
e_{I}^{\mu} C_{\mu J K}=4 \pi G \frac{\gamma^{2}}{\gamma^{2}+1}\left(\frac{1}{2} \epsilon_{I J K L} A^{L}-\frac{1}{\gamma} \eta_{I[J} A_{K]}\right) .
$$

If one does the same computation starting with the action (10) $\tilde{S}_{F}$ one obtains instead

$$
\begin{aligned}
e_{I}^{\mu} C_{\mu J K}= & 4 \pi G \frac{\gamma^{2}}{\gamma^{2}+1}\left(\frac{1}{2} \epsilon_{I J K L}\left(A+\frac{\alpha}{\gamma} V\right)^{L}\right. \\
& \left.-\frac{1}{\gamma} \eta_{I[J}(A-\alpha \gamma V)_{K]}\right) .
\end{aligned}
$$

It is clear from this expression that $e_{I}^{\mu} C_{\mu}{ }^{I K} \propto \gamma /\left(\gamma^{2}+\right.$

\footnotetext{
${ }^{5} \mathrm{We}$ use the identities $\overline{\Gamma \psi}=\bar{\psi} \gamma_{0} \Gamma^{\dagger} \gamma_{0}, \quad\left\{\gamma_{K}, \gamma_{[I} \gamma_{J]}\right\}=$ $-2 i \epsilon_{I J K L} \gamma^{5} \gamma^{L},\left[\gamma_{K}, \gamma_{[I} \gamma_{J]}\right]=4 \eta_{K[I} \gamma_{J]}$.
}

1) $(A-\alpha \gamma V)^{K}$ is different from 0 unless $\gamma=0$ or $\gamma=\infty$. ${ }^{6}$ This shows, as previously stated, that one should take $\alpha$ real in order to have a consistent action principle.

We can now obtain an equivalent action by replacing $\omega$ with $\tilde{\omega}+C(\psi)$ in (5) and (10). The terms of (5) linear in the fermion current are total derivatives, leaving us with the standard second order tetrad action of general relativity with fermions, plus a four-Fermi interaction term $S_{\text {int }}[e, \psi]=S_{\text {int }}^{(1)}[e, \psi]+S_{\text {int }}^{(2)}[e, \psi]$. This interaction term comes first from the evaluation of

$$
S_{\text {int }}^{(1)}[e, \psi]=\frac{1}{16 \pi G} \int d^{4} x e e_{I}^{\mu} e_{J}^{\nu} P_{K L}^{I J}\left[C_{\mu}, C_{\nu}\right]^{K L} .
$$

One can use the following identity

$$
e_{I}^{\mu} e_{J}^{\nu} P_{K L}^{I J}\left[C_{\mu}, C_{\nu}\right]^{K L}=6\left(U^{2}-\frac{2 U V}{\gamma}-V^{2}\right)
$$

if $e_{I}^{\mu} C_{\mu J K}=\epsilon_{I J K L} U^{L}+\eta_{I J} V_{K}-\eta_{I K} V_{J}$, in order to get

$$
S_{\text {int }}^{(1)}[e, \psi]=\frac{3}{2} \pi G\left(\frac{\gamma^{2}}{\gamma^{2}+1}\right)\left(A^{2}+\frac{2 \alpha}{\gamma} A \cdot V-\alpha^{2} V^{2}\right) .
$$

The other contribution is given by

$$
S_{\mathrm{int}}^{(2)}[e, \psi]=\int d^{4} x \frac{e}{4} e_{I}^{\mu} C^{I J K}\left(\epsilon_{I J K L} A^{L}+2 \alpha \eta_{I[J} V_{K]}\right)
$$

leading to the final expression for the effective action

$$
S_{\mathrm{int}}[e, \psi]=-\frac{3}{2} \pi G\left(\frac{\gamma^{2}}{\gamma^{2}+1}\right)\left(A^{2}+\frac{2 \alpha}{\gamma} A \cdot V-\alpha^{2} V^{2}\right) \text {. }
$$

Note that this effective action is similar yet different in detail from the one derived in [7]. One sees that the only interaction which is independent of $\alpha$ is the axial-axial interaction. The parity-violating vector-axial interaction is absent if one considers the minimal coupling $\alpha=0$. Thus parity violation cannot be taken as a measure of the Immirzi parameter but only of the combination $\frac{\alpha \gamma}{\gamma^{2}+1}$ depending on the nonminimal coupling parameter. Note also that when the Immirzi parameter is purely imaginary $\gamma=$ $\pm i$, the interaction becomes infinite. This infinite factor

\footnotetext{
${ }^{6}$ Another theoretical possibility is to have the constraint $A^{K}=$ $\alpha \gamma V^{K}$ satisfied. This is possible if we have $\alpha \gamma= \pm 1$. In this case this constraint is satisfied if all fermions are right handed $(\alpha \gamma=-1)$ or left handed $(\alpha \gamma=-1)$. However this theoretical possibility is clearly excluded by experiments, since we see both right and left handed fermions and we will therefore not discuss this option in what follows. Note that this argument relies on the fact that in our framework the nonminimal coupling $\alpha$ is supposed to be the same for all species. It is logically possible to relax this constraint and study the case of a "speciesdependent" $\alpha$ [9]. We will not study this possibility which seems unnatural.
} 
multiplies the term $(A \mp i \alpha V)^{2}$ and effectively imposes the constraint between vector and axial-vector currents discussed in footnote 2 .

We now turn to the discussion of experimental significance of the gravitational parity violation for both nonminimal and minimal couplings of fermions.

\section{PARITY VIOLATION: THE WEAK CHARGE}

In this section we review the necessary physics needed to discuss possible gravitational parity violation.

We start with the low-energy effective Lagrangian describing interactions between electrons and quarks induced by $Z$ exchange

$$
\begin{aligned}
\mathcal{L}_{\text {eff }}= & -\frac{8 G_{F}}{\sqrt{2}} \rho \sum_{q}\left[g_{L}^{q} \bar{q}_{L} \gamma^{\mu} q_{L}+g_{R}^{q} \bar{q}_{R} \gamma^{\mu} q_{R}\right] \\
& \times\left[g_{L}^{e} \bar{e}_{L} \gamma_{\mu} e_{L}+g_{R}^{e} \bar{e}_{R} \gamma_{\mu} e_{R}\right],
\end{aligned}
$$

where

$$
g_{L}^{f}=I_{f}-Q_{f} \sin ^{2} \theta_{W}, \quad g_{R}^{f}=-Q_{f} \sin ^{2} \theta_{W} .
$$

$I_{f}$ and $Q_{f}$ are, respectively, the isospin and charge of the fermion. The parity-violating part of Eq. (24) is

$$
\begin{aligned}
\mathcal{L}_{P V}= & \frac{G_{F}}{\sqrt{2}} \sum_{q}\left[C_{1 q}\left(\bar{q} \gamma^{\mu} q\right)\left(\bar{e} \gamma_{\mu} \gamma_{5} e\right)\right. \\
& \left.+C_{2 q}\left(\bar{q} \gamma^{\mu} \gamma_{5} q\right)\left(\bar{e} \gamma_{\mu} e\right)\right],
\end{aligned}
$$

where

$$
\begin{aligned}
& C_{1 q}=2 \rho\left(g_{L}^{q}+g_{R}^{q}\right)\left(g_{L}^{e}-g_{R}^{e}\right), \\
& C_{2 q}=2 \rho\left(g_{L}^{q}-g_{R}^{q}\right)\left(g_{L}^{e}+g_{R}^{e}\right) .
\end{aligned}
$$

The first term of Eq. (26) leads to a parity-violating interaction between electrons and nuclei whose amplitude is given by

$$
\begin{aligned}
\mathcal{M}= & \frac{G_{F}}{\sqrt{2}} \sum_{q} C_{1 q} \int d^{4} x\left\langle Z, N\left|\bar{q}(x) \gamma^{\mu} q(x)\right| Z, N\right\rangle \\
& \times\left\langle e^{-}\left(p_{f}\right)\left|\bar{e}(x) \gamma_{\mu} \gamma_{5} e(x)\right| e^{-}\left(p_{i}\right)\right\rangle,
\end{aligned}
$$

where $|Z, N\rangle$ denotes a nucleus consisting of $Z$ protons and $N$ neutrons.

In the nonrelativistic or static limit of the nucleus, we can neglect the space components of the quark vector current matrix element. Its time component

$$
\left\langle Z, N\left|\bar{q}(x) \gamma^{0} q(x)\right| Z, N\right\rangle=\left\langle Z, N\left|q^{\dagger}(x) q(x)\right| Z, N\right\rangle=\rho_{q}(\mathbf{r})
$$

yields the density of quark flavor $q$ inside the nucleus. Since the size of the nucleus can be assumed to be small compared to the wavelength of the momentum transfer $\left(p_{f}-p_{i}\right)$, we can make the approximation

$$
\rho_{q}(\mathbf{r}) \approx N_{q} \delta^{(3)}(\mathbf{r}),
$$

where $N_{q}$ is the total number of quarks of flavor $q$ contained in the nucleus. Therefore,

$$
\begin{aligned}
& \sum_{q} C_{1 q}\left\langle Z, N\left|\bar{q}(x) \gamma^{0} q(x)\right| Z, N\right\rangle \\
& =\left[C_{1 u}(2 Z+N)+C_{1 d}(Z+2 N)\right] \delta^{3}(\mathbf{r}) \\
& \equiv-\frac{1}{2} Q_{W}(Z, N) \delta^{(3)}(\mathbf{r}),
\end{aligned}
$$

where $\left(g_{L}^{e}-g_{R}^{e}\right)=-\frac{1}{2}$ in $C_{1 q}$ is factored out so that $Q_{W}(Z, N)$ depends only on the nucleus.

Next, using the relation between Dirac spinors $u(p)$ (in the Dirac representation) and nonrelativistic Pauli spinors $\phi$,

$$
u(p)=\sqrt{E+m}\left[\begin{array}{c}
\phi \\
\frac{\vec{\sigma} \cdot \vec{p}}{E+m} \phi
\end{array}\right],
$$

we find that the time component of the electron axialvector current matrix element in the nonrelativistic limit becomes

$$
\begin{aligned}
& \left\langle e^{-}\left(p_{f}\right)\left|\bar{e}(x) \gamma_{0} \gamma_{5} e(x)\right| e^{-}\left(p_{i}\right)\right\rangle \\
& =\frac{1}{\sqrt{2 E_{f} V}} \frac{1}{\sqrt{2 E_{i} V}} \bar{u}\left(p_{f}\right) \gamma_{0} \gamma_{5} u\left(p_{i}\right) e^{-i\left(p_{i}-p_{f}\right) x} \\
& =\frac{1}{2 m_{e} V} \phi_{f}^{\dagger}\left[\vec{\sigma} \cdot \vec{p}_{f}+\vec{\sigma} \cdot \vec{p}_{i}\right] \phi_{i} e^{i\left(\mathbf{p}_{i}-\mathbf{p}_{f}\right) \cdot \mathbf{r}} .
\end{aligned}
$$

Inserting Eqs. (31) and (33) into (28), we find

$$
\begin{aligned}
\mathcal{M}= & -\frac{G_{F}}{4 \sqrt{2} m_{e}} Q_{W}(Z, N) \int d^{4} x \varphi_{f}^{\dagger}(x)\left[\hat{\vec{\sigma}} \cdot \hat{\vec{p}} \delta^{3}(\hat{\mathbf{r}})\right. \\
& \left.+\delta^{3}(\hat{\mathbf{r}}) \hat{\vec{\sigma}} \cdot \hat{\vec{p}}\right] \varphi_{i}(x),
\end{aligned}
$$

where

$$
\varphi(x)=\frac{1}{\sqrt{V}} e^{-i p x} \phi
$$

From Eq. (34), we conclude that the first term of Eq. (26) induces a parity-violating potential of the form

$$
\hat{V}_{\mathrm{PV}}=\frac{G_{F}}{4 \sqrt{2} m_{e}} Q_{W}(Z, N)\left[\hat{\vec{\sigma}} \cdot \hat{\vec{p}} \delta^{3}(\hat{\mathbf{r}})+\delta^{3}(\hat{\mathbf{r}}) \hat{\vec{\sigma}} \cdot \hat{\vec{p}}\right],
$$

where $m_{e}, \hat{\vec{\sigma}} / 2, \hat{\vec{p}}$, and $\hat{\vec{r}}$ are, respectively, the mass, spin, momentum, and position of the electron. The factor $Q_{W}(Z, N)$ is called the "weak charge" of the nucleus and can be large enough for heavy nuclei to make the effects of this potential observable.

The second term of Eq. (26) induces a potential dependent on the nuclear spin [10] which is too weak to be observed. 


\section{QUANTUM GRAVITY AND PARITY VIOLATION}

\section{A. Nonminimal coupling}

According to the above discussion (Sec. II) the effective Lagrangian contains a parity-violating term provided the coupling of fermions in the presence of torsion is nonminimal, i.e. $\alpha \neq 0$,

$$
\mathcal{L}_{\mathrm{PV}}=\frac{3}{2} \pi G_{N} \frac{2 \alpha \gamma}{\gamma^{2}+1} e\left(\bar{\psi} \gamma_{A} \psi\right)\left(\bar{\psi} \gamma^{A} \gamma_{5} \psi\right) .
$$

If we allow $\gamma$ to be purely imaginary the overall effective coupling in the low-energy Lagrangian is imaginary $(\alpha$ being real), and thus unitarity is violated. Thus the purely imaginary values of the Immirzi parameter are excluded by appealing to unitarity. Furthermore, the contact interaction blows up at $\gamma= \pm i$. Note that $\gamma=i$ corresponds to the self-dual Ashtekar canonical formalism [7].

Let $\gamma$ be real and let

$$
\frac{2 \gamma}{\gamma^{2}+1} \alpha \equiv \beta, \quad-\infty<\beta<\infty .
$$

Then the above effective interaction becomes

$$
\mathcal{L}_{\mathrm{PV}}=\frac{3}{2} \pi \beta G_{N}\left(\bar{\psi} \gamma_{\mu} \psi\right)\left(\bar{\psi} \gamma^{\mu} \gamma_{5} \psi\right),
$$

which has the same form as the first term of Eq. (26) if we assign the vector current to the quarks and the axial-vector current to the electrons:

$$
\mathcal{L}_{\mathrm{PV}}=\frac{3}{2} \pi \beta G_{N}\left(\bar{q} \gamma_{\mu} q\right)\left(\bar{e} \gamma^{\mu} \gamma_{5} e\right)
$$

Therefore, the parity-violating interaction amplitude is

$$
\begin{aligned}
\mathcal{M}_{\mathrm{PV}}= & \frac{3}{2} \pi \beta G_{N} \sum_{q} \int d^{4} x\left\langle Z, N\left|\bar{q}(x) \gamma^{\mu} q(x)\right| Z, N\right\rangle \\
& \times\left\langle e^{-}\left(p_{f}\right)\left|\bar{e}(x) \gamma_{\mu} \gamma_{5} e(x)\right| e^{-}\left(p_{i}\right)\right\rangle .
\end{aligned}
$$

Using

$$
\begin{aligned}
\left\langle Z, N\left|\bar{q}(x) \gamma^{0} q(x)\right| Z, N\right\rangle & =\left\langle Z, N\left|q^{\dagger}(x) q(x)\right| Z, N\right\rangle \\
& =\rho_{q}(\mathbf{r}) \approx N_{q} \delta^{(3)}(\mathbf{r}),
\end{aligned}
$$

we have

$$
\sum\left\langle Z, N\left|\bar{q}(x) \gamma^{0} q(x)\right| Z, N\right\rangle=3(Z+N) \delta^{(3)}(\mathrm{r}),
$$

and using Eq. (33) we find

$$
\begin{aligned}
\mathcal{M}_{\mathrm{PV}}= & \frac{9 \pi \beta G_{N}(Z+N)}{4 m_{e}} \int d^{4} x \varphi_{f}^{\dagger}(x)\left[\hat{\vec{\sigma}} \cdot \hat{\vec{p}} \delta^{3}(\hat{\mathbf{r}})\right. \\
& \left.+\delta^{3}(\hat{\mathbf{r}}) \hat{\vec{\sigma}} \cdot \hat{\vec{p}}\right] \varphi_{i}(x) .
\end{aligned}
$$

Comparison with the $Z$-exchange case shows that the corresponding coefficients are

$$
-G_{F} Q_{W}(Z, N) \leftrightarrow 9 \pi \sqrt{2} \beta G_{N}(Z+N),
$$

so that the "effective" weak charge for the Immirzi parameter is

$$
Q_{I}=-9 \pi \beta(Z+N)\left(\frac{\sqrt{2} G_{N}}{G_{F}}\right) .
$$

The values of $G_{N}$ and $G_{F}$ are [11]

$$
\begin{aligned}
G_{N} & =6.707(10) \times 10^{-39}(\hbar c)\left(\mathrm{GeV} / c^{2}\right)^{-2}, \\
G_{F} & =1.16639(1) \times 10^{-5}(\hbar c)^{3}(\mathrm{GeV})^{-2} .
\end{aligned}
$$

So in natural units, the ratio of $\sqrt{2} G_{N}$ to $G_{F}$ is about $10^{-33}$. The factor $9 \pi$ is about $3 \times 10^{1}$, and the factor $(Z+N)$ is about $10^{2}$ for heavy nuclei, so the effective weak charge is about $10^{-30} \beta$.

The weak charges of heavy nuclei are typically of order $10^{2}$, and the experimental errors on them are of order 1 [12]. So the experimental constraint on $\beta$ will typically be

$$
\beta \equiv \frac{2 \gamma}{\gamma^{2}+1} \alpha<\text { about } 10^{30}
$$

which is practically no constraint at all. Obviously the physical reason for this is the weakness of gravity as compared to weak interactions, and the fact that parity is already maximally violated in the weak sector.

The crucial point here is that the parity-violating interaction in principle contains two undetermined parameters, the strength of the nonminimal coupling of fermions to gravity and the Immirzi parameter. This seems to have been overlooked in the literature.

\section{B. Minimal coupling}

In this situation $(\alpha=0)$ the effective Lagrangian contains only the axial-axial coupling and the experimental bound on the effective coupling is known in the literature both on four-dimensional [3] and large extra-dimensional physics $[13,14]$. The effective action reads

$$
\mathcal{L}_{\mathrm{AA}}=-\frac{3}{2} \pi G_{N} \frac{\gamma^{2}}{\gamma^{2}+1} e\left(\bar{\psi} \gamma_{A} \gamma_{5} \psi\right)\left(\bar{\psi} \gamma^{A} \gamma_{5} \psi\right),
$$

where we set $\frac{3}{2} \pi G_{N} \frac{\gamma^{2}}{\gamma^{2}+1} \equiv \frac{3 \pi}{\Lambda_{T}^{2}}$ in order to compare with $[13,14]$.

For example, the axial-axial contact four-Fermi interaction can affect the electron-quark contact interactions. A typical bound is $[13,14]$

$$
\Lambda_{T} \geq 5.3 \mathrm{TeV} .
$$

A stronger constraint is implied by astrophysical data provided one assumes the existence of light sterile neutrinos. From supernova data one infers [14]

$$
\Lambda_{T} \geq 210 \mathrm{TeV} \text {. }
$$

If we assume that $\gamma$ is real, then 


$$
\frac{\gamma^{2}}{\gamma^{2}+1}<1
$$

Thus there is no bound on $\gamma$ in this case, given the usual value of the Planck scale.

\section{CONCLUSIONS}

In this paper we have discussed the issue of parity violation in quantum gravity. In particular, we have clarified the role of the coupling of fermionic degrees of freedom in the presence of torsion as well as the physical meaning of the Immirzi parameter in the low-energy effective Lagrangian. The low-energy effective theory is found to contain two parameters: the nonminimal coupling parameter $(\alpha)$ and the Immirzi parameter $(\gamma)$. Only in the case of nonminimal coupling $(\alpha \neq 0)$ the effective Lagrangian contains the axial-vector interaction leading to parity violation. The important point here is that the parity-violating vector-axial interaction contains two parameters $(\alpha$ and $\gamma)$. In this situation, the experimental constraint of an effective parameter involving both $\alpha$ and $\gamma$ can be discussed. In the case of minimal coupling ( $\alpha=$ 0 ) there is no parity violation and the effective Lagrangian contains only the usual axial-axial interaction. Here the bounds on the effective coupling, a function of the Immirzi parameter, are well known in the literature. These do not impose any constraint on the real value of $\gamma$. On the other hand, purely imaginary values of the Immirzi parameter lead to violations of unitarity for $\alpha \neq 0$. Finally, the effective Lagrangian blows up for $\gamma= \pm i$.

\section{ACKNOWLEDGMENTS}

It is our pleasure to thank A. Perez and C. Rovelli for discussions of their work and A. Randono for a correspondence on the issue of a purely imaginary $\gamma$. We also thank R. Jackiw for drawing our attention to his work with S.-Y. Pi. The research of D. M. and T. T. is supported in part by the U.S. Department of Energy under Contract No. DEFG05-92ER40677.
[1] R. Jackiw and S. Y. Pi, Phys. Rev. D 68, 104012 (2003).

[2] S. Holst, Phys. Rev. D 53, 5966 (1996).

[3] For a recent review, please consult I. L. Shapiro, Phys. Rep. 357, 113 (2002) and references therein. A classic review is F. W. Hehl, P. Von Der Heyde, G. D. Kerlick, and J. M. Nester, Rev. Mod. Phys. 48, 393 (1976).

[4] See, for example, N. Arkani-Hamed, H. Georgi, and M. D. Schwartz, Ann. Phys. (N.Y.) 305, 96 (2003); N. ArkaniHamed and M.D. Schwartz, Phys. Rev. D 69, 104001 (2004); V. Jejjala, R. G. Leigh, and D. Minic, Phys. Lett. B 556, 71 (2003); V. Jejjala, R. G. Leigh, and D. Minic, J. Cosmol. Astropart. Phys. 06 (2003) 002; J. de Boer, V. Jejjala, and D. Minic, Phys. Rev. D 71, 044013 (2005).

[5] L. Freidel and D. Minic (work in progress).

[6] L. Freidel and A Starodubtsev, hep-th/0501191.

[7] A. Perez and C. Rovelli, gr-qc/0505081, and references therein.
[8] A. Ashtekar and J. Lewandowski, Classical Quantum Gravity 21, R53 (2004).

[9] A. Randono (private communication).

[10] C. Bouchiat, J. Phys. G 3, 183 (1977).

[11] S. Eidelman et al. (Particle Data Group), Phys. Lett. B 592, 1 (2004).

[12] J. Guena, M. Lintz, and M. A. Bouchiat, Mod. Phys. Lett. A 20, 375 (2005); S. C. Bennett and C. E. Wieman, Phys. Rev. Lett. 82, 2484 (1999).

[13] A. S. Belyaev and I. L. Shapiro, Nucl. Phys. B543, 20 (1999); A. S. Belyaev and I. L. Shapiro, Phys. Lett. B 425, 246 (1998) and references therein.

[14] L. N. Chang, O. Lebedev, W. Loinaz, and T. Takeuchi, Phys. Rev. Lett. 85, 3765 (2000) and references therein. See also, L. N. Chang and C. Soo, Classical Quantum Gravity 20, 1379 (2003); L. N. Chang and C. Soo, Phys. Rev. D 55, 2410 (1997). 